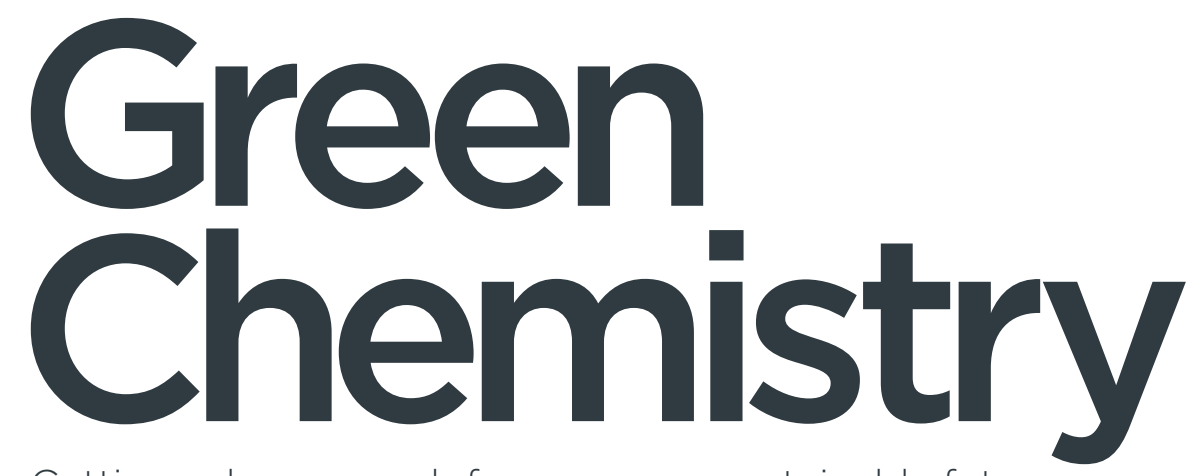

Volume 23

Number 22

21 November 2021

Pages 8711-9182

Cutting-edge research for a greener sustainable future

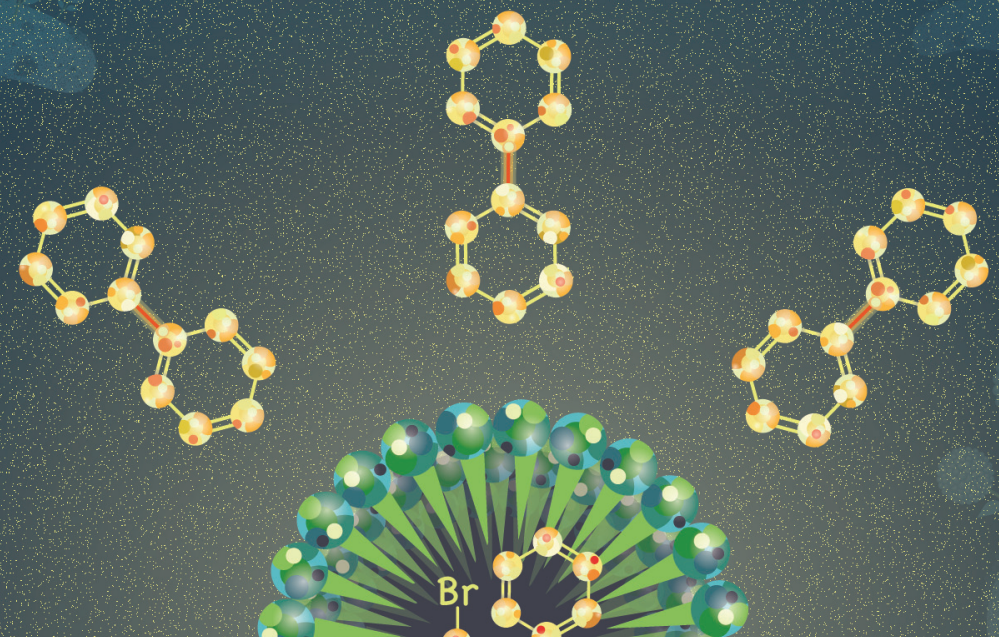

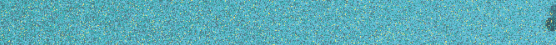

ISSN 1463-9262

ROYAL SOCIETY OF CHEMISTRY

\section{PAPER}

Stephen Wallace, Louise E. Horsfall et al.

Micellar catalysis of the Suzuki Miyaura reaction using

biogenic Pd nanoparticles from Desulfovibrio alaskensis 


\section{D) Check for updates}

Cite this: Green Chem., 2021, 23. 8886

\title{
Micellar catalysis of the Suzuki Miyaura reaction using biogenic Pd nanoparticles from Desulfovibrio alaskensis $\uparrow$
}

\author{
Yuta Era, (D) Jonathan A. Dennis, (D) ${ }^{\text {a,b }}$ Stephen Wallace (D) *a and \\ Louise E. Horsfall (D)*a
}

\begin{abstract}
Microorganisms produce metal nanoparticles (MNPs) upon exposure to toxic metal ions. However, the catalytic activity of biosynthesised MNPs remains underexplored, despite the potential of these biological processes to be used for the sustainable recovery of critical metals, including palladium. Herein we report that biogenic palladium nanoparticles generated by the sulfate-reducing bacterium Desulfovibrio alaskensis G20 catalyse the ligand-free Suzuki Miyaura reaction of abiotic substrates. The reaction is highly efficient ( $>99 \%$ yield, 0.5 mol\% Pd), occurs under mild conditions $\left(37^{\circ} \mathrm{C}\right.$, aqueous media) and can be accelerated within biocompatible micelles at the cell membrane to yield products containing challenging biaryl bonds. This work highlights how native metabolic processes in anaerobic bacteria can be combined with green chemical technologies to produce highly efficient catalytic reactions for use in sustainable organic synthesis.
\end{abstract}

Received 6th July 2021, Accepted 22nd September 2021

DOI: $10.1039 / \mathrm{d} 1 \mathrm{gc} 02392 \mathrm{f}$

rsc.li/greenchem beginning to be explored. Desulfovibrio spp. has the unique capability to generate small nanoparticles in high yield (>95\%) from a variety of feedstocks in the absence of external reductants. The biosynthesis of MNPs also offers distinct advantages over chemically synthesised equivalents from a product uniformity and sustainability viewpoint. Herein we report that bio-

A. Previous work: Micellar catalysis using Pd nanoparticles<smiles>Cc1cc(C)cc(-c2ccc(C(C)(C)C)cc2)c1</smiles>

B. This work: Micellar catalysis using biogenic Pd nanoparticles from Desulfovibrio spp.

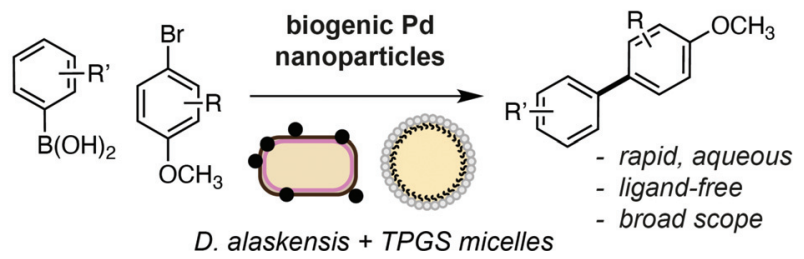

Scheme 1 Approaches to the Suzuki Miyaura cross-coupling reactions in TPGS micelles using chemically synthesised or biogenic palladium nanoparticles. 
genic Pd nanoparticles generated by Desulfovibrio alaskensis G20 are highly active catalysts for the ligand-free Suzuki Miyaura cross-coupling reaction under ambient conditions. The reaction has broad scope, can be dramatically accelerated within membrane associated TPGS micelles and outperforms other heterogeneous NP catalysts generated microbially or via chemical synthesis. Overall, this suggests a unique feature of the metal reduction pathway in Desulfovibrio spp. that is especially suited to the generation of highly active MNP catalysts for use in organic synthesis (Scheme 1).

\section{Results and discussion}

Our studies began by investigating whether bacteriogenic PdNPs from Desulfovibrio alaskensis G20 (DaPdNPs) could catalyse $\mathrm{C}-\mathrm{C}$ bond formation. We chose the Suzuki Miyaura reaction, inspired by previous studies by Deplanche et $a l^{8}{ }^{8}$ who demonstrated the use of E. coli-supported Pd-NPs to catalyse the crosscoupling of aryl halides and phenylboronic acid (62\% conversion, $\left.\mathrm{EtOH}_{(\mathrm{aq})}, 80^{\circ} \mathrm{C}\right)$. To this end, DaPdNPs were prepared from anaerobic cultures of $D$. alaskensis G20 grown in the presence of $\mathrm{Na}_{2} \mathrm{PdCl}_{4}\left(30^{\circ} \mathrm{C}, 20 \mathrm{~h}\right)$ and isolated via centrifugation in $97 \%$ yield, as determined by ICP-OES. Analysis of the nanoparticles by X-ray diffraction (XRD) confirmed the presence of zero-valent Pd in a standard cubic arrangement (ESI, Fig. S2 $\dagger$ ). To test the reactivity of DaPdNPs we used the substrates 4-bromoanisole 1 and phenylboronic acid 2. Reactions were carried out in deionised water at $37^{\circ} \mathrm{C}$ for $20 \mathrm{~h}$ in the presence of ligands that are known to increase the reactivity of $\mathrm{Pd}$ in aqueous media. Encouragingly, 4-methoxybiphenyl 3 was detected in all reactions and also in the absence of any additional ligand (Table 1, entry 1). The ligand-free activity of biogenic MNPs at ambient temperature is rare and suggested to us that DaPdNPs possess unique catalytic properties. No product was observed using 4-chloroanisole and 4-iodoanisole showed no increase in reactivity when compared to $\mathbf{1}$. Interestingly, the addition of triphenylphosphine decreased the cross coupling of 1 and 2 to 24\%, whereas the bis-phenylphosphine ligands JohnPhos 4 and XPhos 6 typically used in Buchwald-Hartwig amination reactions increased the yield to $38 \%$ and $40 \%$, respectively. The water-soluble ligands sSPhos 7 and AmPyol 8 decreased the yield 2-3-fold, despite being widely used to enhance the reactivity of $\mathrm{Pd}$ catalysed reactions in aqueous and/or biological conditions. However, the addition of tetramethylguanidine (TMG, 9) increased the yield of 3 to $62 \%$. As the cell membrane of $D$. alaskensis is negatively charged and tightly associates to Pd-NPs the co-localisation of cationic TMG to the cell surface under the reaction conditions could account for this observation. No further improvement in yield was observed using green tea polyphenols, despite the use of these plant-derived compounds being reported to enable ligand-free cross coupling reactions under aqueous conditions. ${ }^{9}$ Finally, we examined the mechanism of the reaction and compared the activity of biogenic nanoparticles to chemically synthesised equivalents. We confirmed the reaction is heterogeneous using
Table 1 Catalyst, ligand and additive screen for the DaPdNP catalysed Suzuki Miyaura reaction ${ }^{a}$

\begin{tabular}{|c|c|c|c|c|}
\hline & $\begin{array}{l}\mathrm{OCH}_{3} \\
\mathbf{1}\end{array}$ & $\mathrm{B}(\mathrm{OH})_{2}$ & $\begin{array}{c}\text { D. alaskensis } \\
\text { G20 PdNPs } \\
\text { ligand, additive, } \\
\mathrm{K}_{2} \mathrm{CO}_{3} \text { in } \mathrm{H}_{2} \mathrm{O} \\
37^{\circ} \mathrm{C}, 20 \mathrm{~h}, 200 \mathrm{rpm}\end{array}$ & $\mathrm{OCH}_{3} 3$ \\
\hline Entry & Catalyst & Ligand & Additive & Yield $(\% \pm \mathrm{SD})$ \\
\hline 1 & DaPdNP & - & - & 34 \\
\hline 2 & DaPdNP & $\mathrm{PPh}_{3}$ & - & 24 \\
\hline 3 & DaPdNP & JohnPhos & - & 38 \\
\hline 4 & DaPdNP & SPhos & - & 33 \\
\hline 5 & DaPdNP & XPhos & - & 40 \\
\hline 6 & DaPdNP & sSPhos & - & 6 \\
\hline 7 & DaPdNP & AmPyol & - & 19 \\
\hline 8 & DaPdNP & TMG & - & 62 \\
\hline 9 & DaPdNP & - & Green tea polyphenols & 26 \\
\hline $10^{b}$ & DaPdNP & - & - & 0 \\
\hline 11 & cPdNP & - & - & $<1$ \\
\hline 12 & cPdNP & XPhos & - & $<1$ \\
\hline 13 & cPdNP & TMG & - & $<1$ \\
\hline 14 & $\mathrm{Pd} / \mathrm{C}$ & - & - & $<1$ \\
\hline 15 & $\mathrm{Pd} / \mathrm{C}$ & XPhos & - & 2 \\
\hline 16 & $\mathrm{Pd} / \mathrm{C}$ & TMG & - & 5 \\
\hline 17 & DaPdNP & - & TPGS-750-M ${ }^{c}$ & 75 \\
\hline 18 & DaPdNP & - & TPGS- $1000^{c}$ & $>99$ \\
\hline
\end{tabular}

${ }^{a}$ Reactions were performed using $1(25 \mathrm{mM}), 2(30 \mathrm{mM}), \mathrm{K}_{2} \mathrm{CO}_{3}$ $(30 \mathrm{mM})$, Pd catalyst $(0.25 \mathrm{mM})$ and ligand $(2.5 \mathrm{mM})$ in sealed tubes under an atmosphere of air. Product concentrations were determined by ${ }^{1} \mathrm{H}$ NMR spectroscopy relative to an internal standard of TMB. All data shown is an average of three experiments to one standard deviation. ${ }^{b}$ Polymer-supported iodobenzoic acid was used. ${ }^{c} 2 \% \mathrm{w} / \mathrm{vol}$.

a three-phase test by observing no product formation when using polymer-supported iodobenzoic acid (Table 1, entry 10), eliminating the possibility that catalysis occurs via the leaching of soluble Pd from DaPdNPs into solution. Interestingly, 3 was

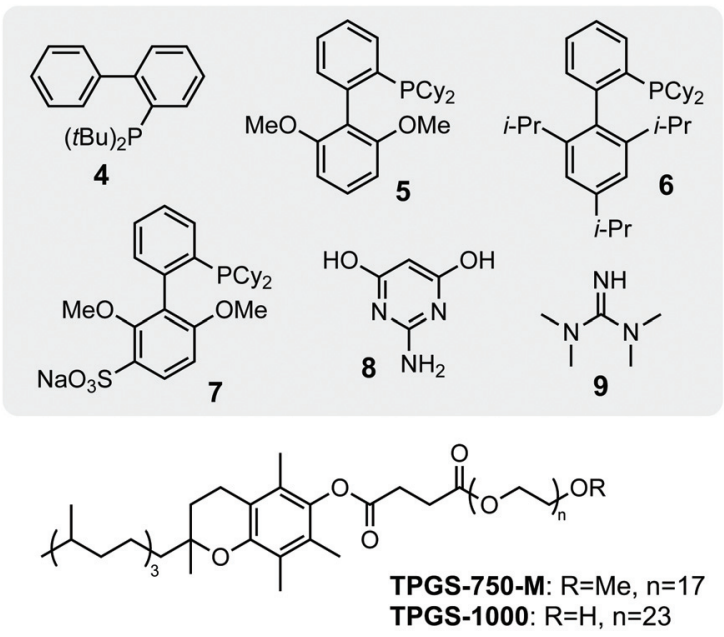

Scheme 2 Structures of $P$ - $/ \mathrm{N}$-ligands and surfactants used in screening studies. JohnPhos 4, SPhos 5, XPhos 6, sSPhos 7, AmPyol 8, TMG 9. 
formed in $<1 \%$ yield when using chemically synthesised heterogeneous Pd catalysts under the same reaction conditions. For example, Pd nanoparticles generated by chemical vapor deposition (cPdNP) and $\mathrm{Pd}$ on activated carbon ( $\mathrm{Pd} / \mathrm{C})$ both afforded 3 in $<1 \%$ yield, increasing to $5 \%$ for $\mathrm{Pd} / \mathrm{C}$ in the presence of 9 (Table 1, entries 11-16). This intriguing result suggests a unique feature of nanoparticle biosynthesis in $D$. alaskensis that is especially suited to the generation of highly active heterogeneous Pd catalysts.

Having confirmed the activity of DaPdNPs under aqueous conditions we moved on to examine methods to increase their
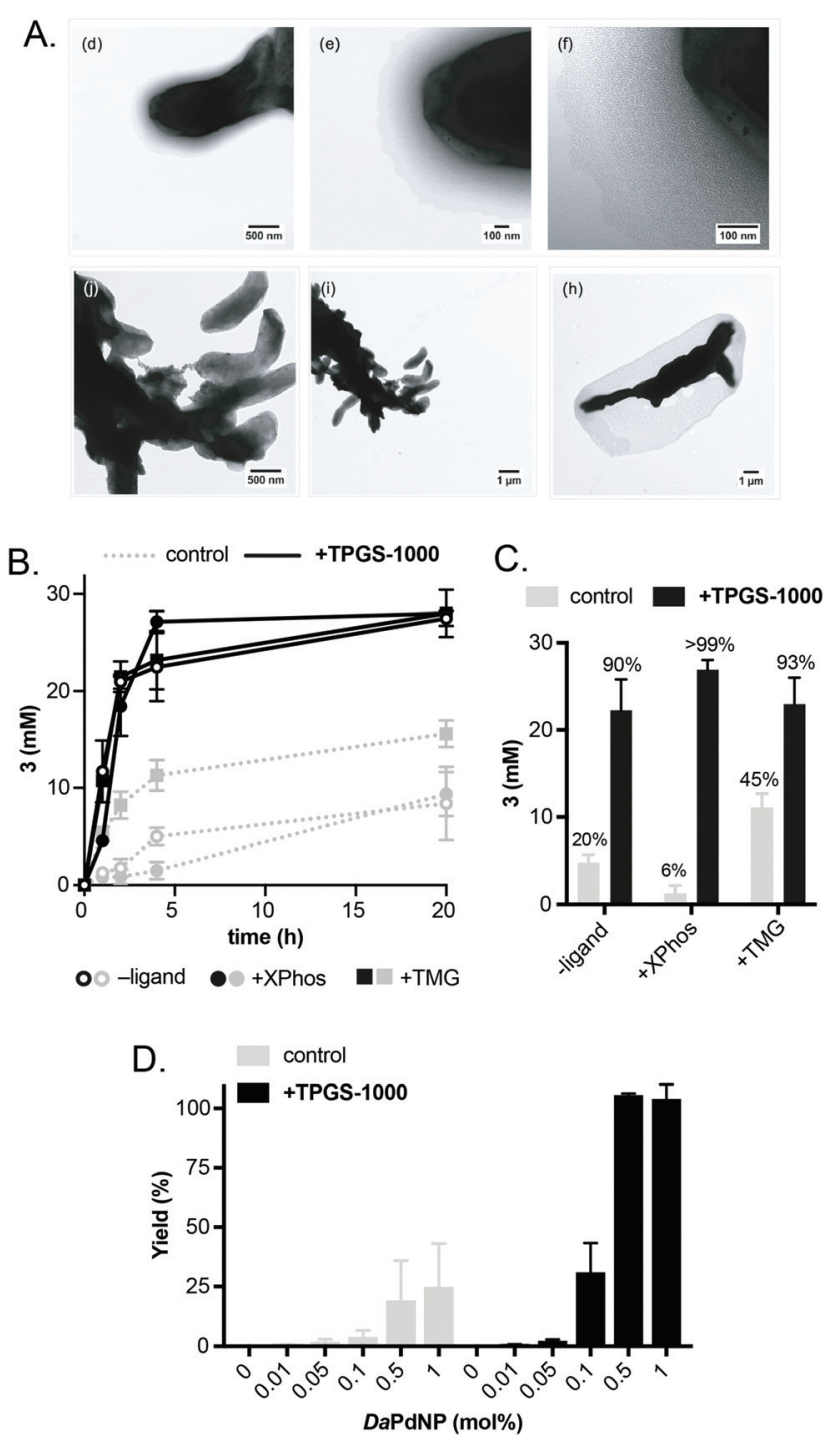

Fig. 1 Examining the effect of TPGS micelles on the reaction. (A) TEM images of DaPdNPs in the presence and absence of TPGS-1000. (B) Time-course analysis showing the production of 3 and the effect of micelle addition. (C) Concentration of 3 in reactions after $4 \mathrm{~h}$. The \% conversion to 3 is shown above each dataset. (D) The effect of micelles on Pd activity at low catalyst loading. $2 \% \mathrm{w} / \mathrm{v}$ TPGS was added in all cases. Pd concentrations were determined by ICP-OES. Error bars represent the standard deviation of values from three independent experiments. activity in vivo. To begin, we examined the effect of co-localising the reaction within TPGS micelles. These vitamin E-derived surfactants are known to self-assemble in aqueous solution and promote organic reactions by co-localising reactants in the hydrophobic micelle interior. ${ }^{10}$ They also associate with cell membranes and accelerate flux through engineered metabolic pathways by sequestering hydrophobic metabolites. ${ }^{11}$ Therefore, as Pd nanoparticles associate to the outer membrane of $D$. alaskensis, we hypothesised that co-localisation of $\mathbf{1}$ and $\mathbf{2}$ to this region would also accelerate product formation. To our delight, the addition of $2 \% \mathrm{w} / \mathrm{v}$ TPGS-750-M and TPGS-1000 significantly increased the reactivity of DaPdNPs in the absence of ligand, affording 3 in $75 \%$ and $>99 \%$ yield, respectively. Increased product formation was observed for all combinations of surfactant and ligand, except for the use of 4 and TPGS-750-M (ESI, Table S3, $\uparrow$ entry 3). In all cases, the hydroxylated surfactant TPGS-1000 outperformed the $O$-methylated congener TPGS-750-M. To explore the reasons for this, we examined cells by transmission electron microscopy (TEM). Interestingly, the addition of TPGS-1000 produced highly-ordered micelles at the cell surface (Fig. 1A and ESI, Fig. S7†), whereas TPGS-750-M produced disordered agglomerates that appeared to disrupt cell morphology (ESI, Fig. S8 $\dagger$ ). Although the increased membrane penetration of TPGS-750-M has been observed in E. coli and attributed to the increased hydrophobicity of the micelle surface (Scheme 2, $\mathrm{R}=$ $\mathrm{CH}_{3}, n=17$ ), this was shown to have no effect on the rate of reactions in the micelle interior. ${ }^{11}$ For $D$. alaskensis, however, TPGS-750-M micelles embedded in the cell membrane are distanced from MNPs bound at the cell surface and could therefore explain the reduced yield of 3. Extracellular presentation of DaPdNPs could also combine with favourable hydrogen bonding interactions between TPGS-1000 (Scheme 2, $\mathrm{R}=\mathrm{H}, n$ = 23) and outer-cell matrix polysaccharides surrounding the Pd nanoparticles to co-localise the substrates and catalyst. Altogether, this provides evidence to support the hypothesis that TPGS-1000 and TPGS-750-M enhance the reactivity of biogenic $\mathrm{Pd}$ nanoparticles from $D$. alaskensis by localising the reaction components at the outer cell membrane. The precise nature of this interaction and the generality of this effect across various microorganisms is underexplored and currently under investigation by our laboratories. To further assess the extent to which TPGS micelles accelerate the reaction we moved on to measure product formation over time (Fig. 1B). In the absence of TPGS-750-M or TPGS-1000, the reaction reached 40 and $60 \%$ conversion after $20 \mathrm{~h}$ in the presence of ligand 6 or 9, respectively. In contrast, TPGS-1000 accelerated the formation of 3 to $90 \%$ after $4 \mathrm{~h}$ in the absence of ligand and to $>99 \%$ and $93 \%$ yield using 6 and 9, respectively (Fig. 1C). High reactivity was also maintained at low catalyst loading. For example, reducing DaPdNPs to $1 \mathrm{~mol} \%$ reduced product conversion to $25 \%$ in the absence of micelles, whereas quantitative conversion was observed in the presence of TPGS-1000 at catalyst loadings of $\geq 0.5 \mathrm{~mol} \%$ (Fig. 1D). This reduced to $31 \%$ and $4 \%$ yield at $0.1 \mathrm{~mol} \% \mathrm{Pd}$ in the presence and absence of TPGS-1000, respectively. Overall, this dramatic 
effect ( $>5$-fold) highlights the unique benefits of micellar catalysis in $D$. alaskensis cells and how this can be combined with organic ligands designed for use in synthetic chemistry to enhance the reactivity of biological metals in vivo.

Using these optimised conditions, we next investigated the reaction scope using a range of aryl bromide and aryl boronic acid substrates (Fig. 2). This included arenes containing a range of electron withdrawing and donating function groups at ortho-, meta- and para-positions and included the heterocyclic (methoxy)pyridyl boronic acid (MeOPyB, 16). To our delight, DaPdNPs were effective catalysts for most substrates tested and when combined with TPGS-1000 enabled the formation of challenging $\mathrm{sp}^{2} \mathrm{C}-\mathrm{C}$ bonds. Electron withdrawing acyl groups were tolerated at all positions in the aryl bromide substrate. Reactivity was reduced for ortho-acyl and ortho-ethoxy substrates 12 and 19 due to steric hindrance, but could be increased through the addition of TPGS-1000. For the cross coupling of $\mathbf{1 1}$ and 16, 12 and 17, 12 and 19, 13 and 16 and 14 and 16, the use of TPGS-1000 increased product yield $>2$-fold. This is particularly impressive for heteroaromatic substrates and the crosscoupling of two ortho-substituted aromatics. Notably, 14 and 19 were unreactive in the presence of DaPdNPs but product formation was increased to $42 \%$ in the presence of TPGS-1000 micelles. Overall, this shows that TPGS micelles not only accelerate product formation in the presence of cells but also increase the overall reaction scope of biogenic PdNPs.

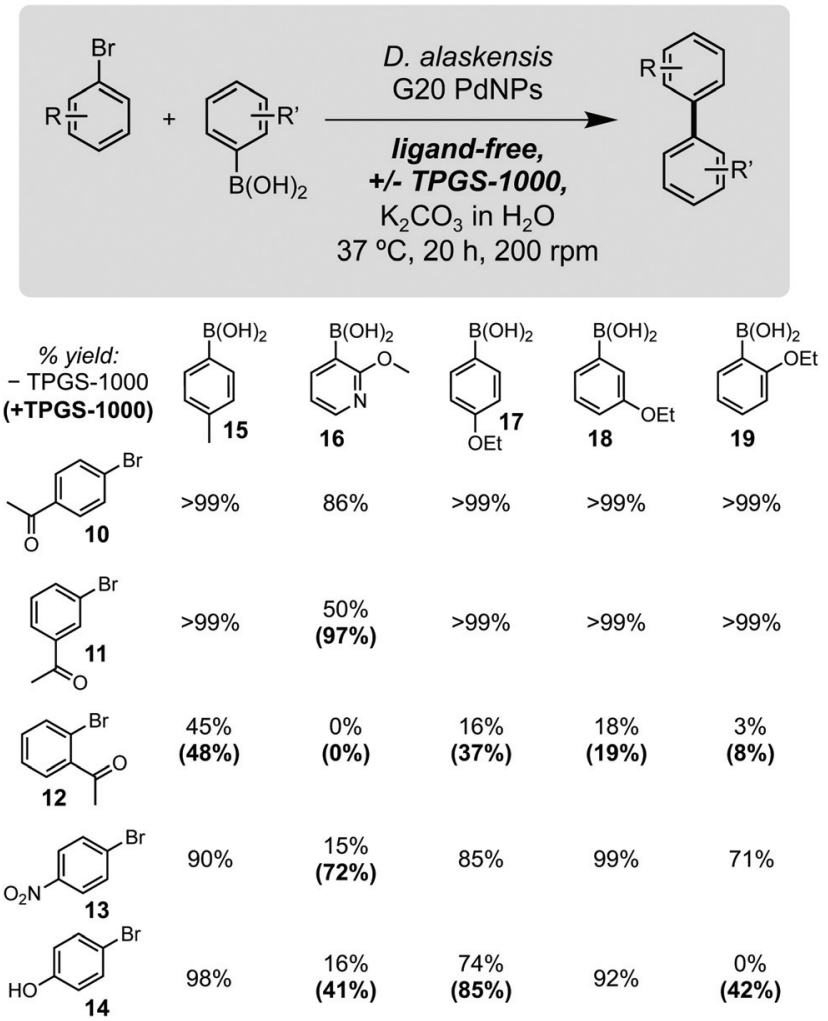

Fig. 2 Substrate scope. Yields in parenthesis are from reactions containing $2 \% \mathrm{w} / \mathrm{v}$ TPGS-1000. Product concentrations determined by ${ }^{1} \mathrm{H}$ NMR spectroscopy relative to an internal standard of TMB (10 mM).

\section{Conclusions}

In summary, we have demonstrated that $\mathrm{Pd}^{0}$ nanoparticles synthesised by Desulfovibrio alaskensis are highly active heterogeneous catalysts for the Suzuki Miyaura reaction of aryl bromides and phenylboronic acids. These biological catalysts can be readily prepared from bacterial cell culture using common Pd salts in high yield (>97\%) and outperform other available heterogenous Pd catalysts generated via chemical or biological methods. We show that reactions catalysed by these nanoparticles can be enhanced using organic ligands and/or designer micelles to co-localise substrates at the cell membrane. The combination of organic chemistry and microbiology tools results in an overall reaction that is highly efficient (>99\% yield, $0.5 \mathrm{~mol} \% \mathrm{Pd}$ ), occurs under ambient conditions (aqueous media, $37^{\circ} \mathrm{C}$ ) and can be applied to a wide range of substrates to generate products containing challenging $\mathrm{sp}^{2} \mathrm{C}-\mathrm{C}$ bonds. To the best of our knowledge, this is the first report of a ligand-free Suzuki Miyaura reaction catalysed by a biological Pd catalyst in micellar nanoreactors. Future studies will focus on genetically engineering D. alaskensis to produce "designer NPs" with increased reactivity and extending this process to encompass the use of industrial waste streams as a metal resource.

\section{Author contributions}

L. E. H. and S. W. conceived and directed the project. Y. E. and J. A. D. planned and conducted experimental work. All authors contributed to discussions and manuscript writing.

\section{Conflicts of interest}

There are no conflicts to declare.

\section{Acknowledgements}

This work was supported by EPSRC fellowship (EP/N026519/1) awarded to L. E. H. Y. E. acknowledges support from the University of Edinburgh (Edinburgh Global Research Scholarship) and Japan Student Services Organization (JASSO) (ND1910900104). J. A. D. acknowledges a PhD studentship from the EPSRC Centre for Doctoral Training in Critical Resource Catalysis (CRITICAT) (EP/L016419/1). S. W. acknowledges a Future Leaders Fellowship from UKRI (MR/S033882/1). The authors thank Dr Tatsuya Tokunaga (Kyushu Institute of Technology) for XRD analysis. We would like to thank Steve Mitchell for his help with the TEM as part of the Wellcome Trust Multi User Equipment Grant (WT104915MA) and Lorna J. Eades for help with the ICP-OES, both at the University of Edinburgh. 


\section{References}

1 (a) The European Commission, Study on the review of the list of Critical Raw Materials, 2017, DOI: 10.2873/876644; (b) D. Chattopadhyay, Resonance, 2017, 22, 79; (c) K. Khan and S. D. Köseoğlu, Resour. Policy, 2020, 68, 101780.

2 (a) R. Panda, M. K. Jha and D. D. Pathak, in Rare Metal Technology 2018, ed. H. Kim, B. Wesstrom, S. Alam, T. Ouchi, G. Azimi, N. R. Neelameggham, S. Wang and X. Guan, Springer International Publishing, 2018, p. 119; (b) M. Bihani, T. N. Ansari, J. D. Smith and S. Handa, Curr. Opin. Green Sustain. Chem., 2018, 11, 45; (c) H. Dong, J. Zhao, J. Chen, Y. Wu and B. Li, Int. J. Miner. Process., 2015, 145, 108; (d) T. Hennebel, B. De Gusseme, N. Boon and W. Verstraete, Trends Biotechnol., 2009, 27, 90; (e) M. K. Jha, J. Lee, M. Kim, J. Jeong, B. Kim and V. Kumar, Hydrometallurgy, 2013, 133, 23; (f) A. Paiva, Metals, 2017, 7, 505.

3 (a) N. J. Creamer, V. S. Baxter-Plant, J. Henderson, M. Potter and L. E. Macaskie, Biotechnol. Lett., 2006, 28, 1475; (b) D. Gauthier, L. S. Søbjerg, K. M. Jensen, A. T. Lindhardt, M. Bunge, K. Finster, R. L. Meyer and T. Skrydstrup, ChemSusChem, 2010, 3, 1036; (c) N. I. Hulkoti and T. C. Taranath, Colloids Surf., B, 2014, 121, 474; (d) L. E. Macaskie, I. P. Mikheenko, P. Yong, K. Deplanche, A. J. Murray, M. Paterson-Beedle, V. S. Coker, C. I. Pearce, R. Cutting, R. A. D. Pattrick, D. Vaughan, G. van der Laan and J. R. Lloyd, Hydrometallurgy, 2010, 104, 483; (e) M. Martins, A. Assunção, H. Martins, A. P. Matos and M. C. Costa, J. Chem. Technol. Biotechnol., 2013, 88, 2039; $(f)$ A. J. Murray, J. Zhu, J. Wood and L. E. Macaskie, Miner. Eng., 2017, 113, 102.

4 (a) G. Cabrera, R. Perez, J. M. Gomez, A. Abalos and D. Cantero, J. Hazard. Mater., 2006, 135, 40; (b) M. J. Capeness, M. C. Edmundson and L. E. Horsfall, New Biotechnol., 2015, 32, 727.

5 (a) L. J. Hauser, M. L. Land, S. D. Brown, F. Larimer, K. L. Keller, B. J. Rapp-Giles, M. N. Price, M. Lin, D. C. Bruce, J. C. Detter, R. Tapia, C. S. Han, L. A. Goodwin, J. F. Cheng, S. Pitluck, A. Copeland, S. Lucas, M. Nolan, A. L. Lapidus, A. V. Palumbo and J. D. Wall, J. Bacteriol., 2011, 193, 4268; (b) J. V. Kuehl, M. N. Price, J. Ray,
K. M. Wetmore, Z. Esquivel, A. E. Kazakov, M. Nguyen, R. Kuehn, R. W. Davis, T. C. Hazen, A. P. Arkin and A. Deutschbauer, mBio, 2014, 5, e01041; (c) M. N. Price, J. Ray, K. M. Wetmore, J. V. Kuehl, S. Bauer, A. M. Deutschbauer and A. P. Arkin, Front. Microbiol., 2014, 5, 577; (d) J. D. Wall, B. J. Rapp-Giles and M. Rousset, J. Bacteriol., 1993, 175, 4121.

6 (a) M. J. Capeness, V. Echavarri-Bravo and L. E. Horsfall, Front. Microbiol., 2019, 10, 997; (b) M. J. Capeness, L. Imrie, L. F. Mühlbauer, T. Le Bihan and L. E. Horsfall, Microbiology, 2019, 165, 1282.

7 (a) M. G. Kiran, K. Pakshirajan and G. Das, J. Environ. Manage., 2018, 218, 486; (b) S. K. Hwang and E. H. Jho, Sci. Total Environ., 2018, 635, 1308; (c) I. H. Kim, J. H. Choi, J. O. Joo, Y. K. Kim, J. W. Choi and B. K. Oh, J. Microbiol. Biotechnol., 2015, 25, 1542; (d) M. G. Kiran, K. Pakshirajan and G. Das, J. Hazard. Mater., 2017, 324, 62; (e) M. Martins, M. L. Faleiro, R. J. Barros, A. R. Verissimo, M. A. Barreiros and M. C. Costa, J. Hazard. Mater., 2009, 166, 706; (f) M. Zhang, H. Wang and X. Han, Chemosphere, 2016, 154, 215.

8 K. Deplanche, J. A. Bennett, I. P. Mikheenko, J. Omajali, A. S. Wells, R. E. Meadows, J. Wood and L. E. Macaskie, Appl. Catal., B, 2014, 147, 651.

9 H. Veisi, A. Rostami and M. Shirinbayan, Appl. Organomet. Chem., 2017, 31, e3609.

10 (a) B. H. Lipshutz, S. Ghorai, A. R. Abela, R. Moser, T. Nishikata, C. Duplais, A. Krasovskiy, R. D. Gaston and R. C. Gadwood, J. Org. Chem., 2011, 76, 4379; (b) F. Gallou, N. A. Isley, A. Ganic, U. Onken and M. Parmentier, Green Chem., 2016, 18, 14; (c) S. Handa, Y. Wang, F. Gallou and B. H. Lipshutz, Science, 2015, 349, 1087; (d) M. CortesClerget, N. Akporji, J. Zhou, F. Gao, P. Guo, M. Parmentier, F. Gallou, J. Y. Berthon and B. H. Lipshutz, Nat. Commun., 2019, 10, 2169; (e) T. Kitanosono, K. Masuda, P. Xu and S. Kobayashi, Chem. Rev., 2018, 118, 679; $(f)$ S. Wallace and E. P. Balskus, Angew. Chem., Int. Ed., 2015, 54, 7106; (g) S. C. Cosgrove, M. P. Thompson, S. T. Ahmed, F. Parmeggiani and N. J. Turner, Angew. Chem., Int. Ed., 2020, 59, 18156.

11 S. Wallace and E. P. Balskus, Angew. Chem., Int. Ed., 2016, 55, 6023 . 